\title{
PESTICIDE RESIDUES IN SOME SELECTED POND WATER SAMPLES OF MEHERPUR REGION OF BANGLADESH
}

\author{
M. AMIN UDDIN, ${ }^{1}$ MINAKSHI SAHA ${ }^{2}$, M.A.Z. CHOWDHURY AND M A. RAHMAN \\ Institute of Food and Radiation Biology, Atomic Energy Research Establishment, \\ Gonakbari, Savar, GPO Box-3787, Dhaka- 1000 \\ ${ }^{2}$ Dept. of Biotechnology and Genetic Engineering, Islamic University, Kushtia
}

\begin{abstract}
Water samples collected from some selected ponds of Meherpur region were studied for the presence of organophosphorus and carbamate pesticide residues. High performance liquid chromatography (HPLC) technique was used to determine the concentration levels of those residues. The results obtained indicate slight contamination of some of the water samples with residues of diazinon, chlorpyriphos (organophosphorus insecticide) and carbofuran (carbamate insecticide). The residues level of diazinon and chlorpyriphos ranged from 0.033 to $0.079 \mathrm{ppm}$ and 0.010 to $0.471 \mathrm{ppm}$ respectively. Among carbamate pesticides, carbofuran identified in two samples ranged from 0.0143 to $0.0387 \mathrm{ppm}$, and carbaryl was not detected in any of the samples. Limit of detection (LOD) was $0.01 \mathrm{ppm}$. However, the residue level was also within the acceptable ranges according to the WHO guideline value of water quality. But, the presence of such residues is indicative of weak regulatory control which is required to be in place for safety of the environment and to stop further aggravation of the situation.
\end{abstract}

Key words: Pesticide, Residue, Organophosphorus, Carbamate, Pond water

\section{Introduction}

The economy of Bangladesh is mainly based on agriculture. So, agriculture is her economic backbone with the production accounting for about one third of the gross domestic product. In Bangladesh, $40 \%$ of the crop loss can be attributed to attack by pests and insects which is a significant loss (Bagchi et. al. 2008). The widely cultivated high yielding variety is highly vulnerable to pests and diseases. So the use of pesticide is now an inherent part of agriculture for pest control. Thus, agrochemicals, including pesticides are considered a critical aid in improving agricultural production and the prevention of crop losses daring pre and post harvest (Rahman et al. 1995).

Due to use of pesticides for agriculture the pond water around the paddy fields may get contaminated. Although pesticide is beneficial for pest control but it also poses a harmful effect to our environment such as the pollution of pond water. After application of pesticide in the crop field the pesticide is degraded in the soil by the soil microorganisms in some extent but many of the toxic pesticide is transported into surface and ground water by agricultural run off rain water from the crop field. Ultimately, the surface and ground water might be highly contaminated due to this agricultural run off

${ }^{1}$ Corresponding author: Email: aminifob@yahoo.com 
pesticide (Bagchi et al. 2008). Use of pesticides has to be controlled to avoid contamination of food supplies and ecological imbalance, but present measures taken in Bangladesh are inadequate and farmers rarely implement standards, allowing sale of unregistered pesticides and misuse (Matin et al. 1998).

Apart from being occupationally hazardous, indiscriminate use of pesticides in developing world is today posing a serious threat to human health. So, in this connection, monitoring pesticide residues is one of the most important aspects in minimizing potential hazards to human health (Bagchi et al. 2008). So, the levels of pesticide residues in various environmental samples should be monitored routinely and effective measures must be adopted to control the use of pesticides for saving the surface water as well as for minimizing human health hazards. Hence, the present study was undertaken to investigate the extent of contamination from pesticide residues (i.e. organophosphorus and carbamate) and their concentration levels in water samples of different locations and propose a sound recommendation for minimizing the pesticide contamination of pond water in those locations of Bangladesh.

\section{Materials and Methods}

Reagents: During this investigation analytical grade hexane (Merck, Germany); acetone (extra pure, BDH, England); anhydrous Sodium Sulphate (Merck,Germany); Florisil ( Magnesium Silicate, Sigma,USA, mesh 60-100) and diethyl ether( analytical grade) were used.

Equipment: Rotary vacuum evaporator (Type -350, USA); High Performance Liquid Chromatograph (HPLC, Waters), Detector-Waters 486 and Pump- Waters 515 were used.

Operating condition of HPLC (UV mode): Detector:Ultra Violet Detector (Fixed wave length), Column: $\mathrm{C}_{18}$, Flow rate: $0.5 \mathrm{~mL} / \mathrm{min}$, Mobile phase: Acetonitrile: Water (65: 35), Wave length : $254 \mathrm{~nm}$, Injection volume: $20 \mu \mathrm{L}$.

Collection and Preservation of water samples: Water samples were collected from some selected ponds of Meherpur area of Bangladesh. Samples were then taken to the laboratory as quickly as possible in glass containers and kept in freezing condition until extraction to avoid degradation.

Extraction of Water: Extraction of water sample (500 mL each) was performed with 100 $\mathrm{mL}$ double distilled hexane in a separatory funnel with shaking for 5 minutes. Hexane extract was separated and collected in evaporating flasks. Two further extractions with $25-\mathrm{mL}$ hexane were done. The combined hexane extract was treated with 5-g anhydrous sodium sulphate to remove traces of water. The water free extract was evaporated to a small volume (Approx. $1 \mathrm{~mL}$ ) and transferred to a glass-Stoppard test tube followed by complete evaporation of solvent (About to dried sample) under a mild stream of nitrogen.

Clean up: The extract was subjected to clean-up using florisil column chromatography (DFG Manual of Pesticide Residue Analysis, 1987). The top $1.5 \mathrm{~cm}$ of the florisil 
column was packed with anhydrous sodium sulphate. Elution was done with $2 \%$ diethyl ether in hexane $(5 \mathrm{~mL} / \mathrm{min})$. The eluate was concentrated in a rotary vacuum evaporator and transferred to glass-stoppard test tubes. Solvents were completely removed under mild nitrogen flow. The evaporated sample was dissolved in acetonitrile and then made to volume $1 \mathrm{~mL}$ in a volumetric flask for high performance liquid chromatography (HPLC).

Sample Analysis: Injections of the aliquots (usually $20 \mu \mathrm{L}$ ) were done by micro syringe into HPLC. Identification of the pesticide was carried out in relation to the retention time of the pure analytical standard supplied by International Atomic Energy Agency (IAEA). Quantification was made with a standard curve of the relevant (standard) pesticide and $\mathrm{r}^{2}=.0 .978345$.

Analysis was done by HPLC (Detector-Waters 486, Pump- waters 515), which was used for the detection of organophosphorus, chlorpyriphos and carbamate pesticide residues.

Chromatographic Determination: For pesticides residues analysis, aliquot was injected by microlitre syringe into the High Performance Liquid chromatography (HPLC) fitted with UV Detector. The wave length was fixed at $254 \mathrm{~nm}$ for organophosphorus and carbamate pesticides.

Extraction efficiency / recovery: Analytical procedures employed were found to be satisfactory and average recoveries between 71 and $93 \%$ were obtained for diazinon, chlorpyriphos and carbofuran pesticides from the water samples (the fortifications were made in the concentration range 0.02-0.2 ppm level) indicating the suitability of the methodology.

\section{Results and Discussion}

Twenty water samples collected from the most vulnerable sites of Meherpur region of Bangladesh were analyzed for the presence of pesticide residues and the data were compared with the results of FAO/WHO recommended level. According to the results of this study, some water samples were found to be contaminated with organophosphorus (diazinon, chlorpyriphos) and carbamate (carbofuran, carbaryl) insecticide residues to some extent. Among the organophosphate pesticides, malathion, chlorpyriphos and diazinon were analyzed. Malathion was not detected in any of the analyzed samples during the present investigation. But, diazinon was found to be present in three water samples; one from Amzhupi union (WS-01; $0.0328 \mathrm{ppm}$ ), another from Kutubpur union (WS-11; $0.0790 \mathrm{ppm}$ ) and the last one from Amdaho union (WS-19; $0.0775 \mathrm{ppm}$ ). The residue of chlorpyriphos was detected in only two samples, one from Kutubpur union (WS-11; $0.0107 \mathrm{ppm}$ ), and another from Amdaho union (WS-19; $0.0143 \mathrm{ppm}$ ). The results are presented in Table 1 . This result matches the survey data which suggest the widespread use of diazinon and chlorpyriphos by the farmers of areas under 
investigation. Because, as a background study, the farmers of the study area informed that they did not apply any organochlorine pesticide but a lot of organophosphate (diazinon and chlorpyriphos) and carbamate pesticides have been applied by them in the cultivating areas. Similar findings were reported by Fatta et al. (2007) in their study.

Table 1. Amount of organophosphorus (OP) and carbamate pesticide residues in pond water samples.

\begin{tabular}{ccccc}
\hline \multirow{2}{*}{$\begin{array}{l}\text { Sample } \\
\text { No }\end{array}$} & $\begin{array}{c}\text { OP pesticide residues in water sample } \\
(\mathrm{ppm})\end{array}$ & \multicolumn{2}{c}{$\begin{array}{c}\text { Carbamate pesticide residues in water } \\
\text { sample (ppm) }\end{array}$} \\
\cline { 2 - 5 } & Diazinon & Chlorpyriphos & Carbofuran & Carbaryl \\
\hline WS1 & 0.0328 & ND & ND & ND \\
WS11 & 0.0790 & 0.0107 & 0.0387 & ND \\
WS19 & 0.0775 & 0.0143 & 0.0143 & ND \\
\hline
\end{tabular}

$\mathrm{W}=$ Water, $\mathrm{S}=$ Sample, ND $=$ Not Detected.

Matin et al. (1998) stated that DDT, DDE and dieldrin were present in some surface water samples obtained from the irrigated crop fields at Gaibandha. Groundwater samples from Nayarhat (hand tube well) were apparently free from residues. In most cases, residual levels were found to be within WHO guideline values for drinking water quality (WHO 1993). However, water samples from a irrigated crop field of Begumganj were found to contain DDT residues at $19 \mu \mathrm{g} / \mathrm{L}$, which was above the WHO guideline value (Matin et al.1998). It was reported that since, Bangladesh is an agro-based country, huge number of pesticides especially carbamate and organophosphorus are used regularly in large quantities in the crop fields. Twenty insecticides, 18 fungicides and 2 rodenticides are being used in our agricultural and public health sectors (Sattar 1985). In the coastal region, large amount of various pesticides are used in the paddy fields, dry fish preservation and horticulture and in betel leaf cultivation. Those pesticides are washed down to the nearby canals, tributaries, rivers and ultimately find their way into the coastal water (Islam et. al. 2001). It is reported that $25 \%$ of the total amount of pesticides used in Bangladesh may reach the coastal water and pollute the sea (Khan and Talukder 1993). Also, Shoeb et al. (2009) reported in a study that organochlorine pesticides as DDTs $(0.432 \mathrm{ppm})$ were found in dry fish used as feed ingredient from shrimp cultivation areas of Bagerhat.

Among the carbamate group, carbofuran was identified in two water samples; one from Kutubpur union (WS-11) and another from Amdaho union (WS-19) and the concentration level of carbofuran was $0.0387 \mathrm{ppm}$ and $0.0143 \mathrm{ppm}$ respectively. The results are presented in Table 1. However, residual levels of carbofuran in all the samples were fairly below the WHO guide line values. But, in a study, Bagchi et al. (2008) observed the highest concentration of carbofuran residue $(2.208 \mathrm{ppm})$ in pond water samples of Bangladesh. Carbaryl was not present in any environmental water samples in 
the present study. This suggests that both carbofuran and carbaryl might not frequently be used by the local farmers of the selected areas and if used, those might be used in a well-controlled manner.

Carbamate pesticides are rapidly taken up by plants through the roots from soil and water and are translocated mainly into the leaves. The main metabolite in plants has been identified as 3-hydroxycarbofuran (Thapar et al.1995). Field studies have indicated a half-life of 26 to 110 days in soil. Carbamate pesticides are degraded in water by hydrolysis, microbial decomposition and photolysis (WHO 2003 and Thapar et al. 1995).

The present findings are relatively less than the findings of others in terms of organophosphorus in water (Sanghi and Sarma 2005). But, the presence of such compounds indicates lapses in regulatory control, which endanger the environment. Although the Pesticide Rule, 1985 of Bangladesh strictly prohibited any kind of unauthorized use of pesticides but because of lack of strong monitoring and proper evaluation facilities this regulation did not work effectively to control indiscriminate use of hazardous pesticides.

\section{References}

Bagchi S., A.K. Azad, M.Alamgir, Z. Chowdhury, M. Amin Uddin, Sharif M. Al-Reza and A. Rahman. 2008. Quantitative Analysis of Pesticide Residues in some Pond water samples of Bangladesh. Asian Journal of water, Environment and Pollution, 6 (4): 27-30.

DFG Manual of Pesticide Residue Analysis. 1987. Pesticide Commission, Weinheim, New York, NY: VCH. 1: 297-307.

Fatta, D., St. Canna-Michaelidou, C, Michael. and E. Demetriou Georgiou. 2007. Organochlorine and organophosphoric insecticides, herbicides and heavy metals residue in industrial wastewaters in Cyprus. Journal of Hazardous Materials 145:169-179.

Islam, M. S., Y. S. A. Khan and S. R. Choudhury. 2001. Effects of organophosphorous pesticides on the behavioral pattern and mortality of Apocryptes bato (Bellder, 1874). Bangladesh J. Zool. 29 (2): 151-157.

Khan, Y.S.A. and A.B.M.A.Talukder.1993. Pollution in coastal water of Bangladesh. Journal of Noami. 10: 7-8.

Matin, M. A., M.A. Malek, M.R. Amin, S. Rahman, J. Khatoon, M. Rahman, M. Amin Uddin and A.J. Miah. 1998. Organochlorine insecticide residues in surface and underground water from different regions of Bangladesh. Agriculture, Ecosystems and Environment. 69: $11-15$.

Rahman, M.S., M.A. Malek and M.A. Matin. 1995. Trend of pesticide usages in Bangladesh. Sci. Total Environ. 159: 35-39.

Sanghi, R and Sarma, A.K.. 2005. Organochlorine and organophosphorus pesticide residues in ground water and surface waters of Kanpur, Uttar Pradesh, India, Environment International, 31 (1) : 113-120.

Sattar, M. A. 1985. Use of the pesticides in Bangladesh and protection of environment. Proceedings of SAARC seminar on protection the environment from degradation, Dhaka. pp. 47-51. 
Shoeb, M., N., Nilufar, M., Shankar H. M. Sarma and M. S. I. Seraji. 2009. Nitrofuran antibiotics and organochlorine pesticides in fish and poultry feed, feed ingredients and water samples collected from shrimp cultivation areas. Journal of Bangladesh chemical Society. 22(1): 1-8.

Thapar, S., R. Bhushan, and R.P. Mathur. 1995. Degradation of organophosphprus carbamate pesticides in soils: HPLC determination. Biomed Chromatography. 9(1):18-22.

WHO (World Health Organization). 2003. Carbofuran in drinking water. Background document for preparation of WHO guidelines for drinking water quality (WHO/SDE/WSH/03.04/81, pp.01). Geneva, World Health Organization.

WHO (World Health Organization). 1993. Guidelines for drinking water quality. 2(2): 940-949.

(Received revised manuscript on 11 May 2013) 